\title{
Caracterização e desempenho logístico das indústrias laticinistas da Zona da Mata e Campo das Vertentes em Minas Gerais, Brasil
}

\author{
Characterization and logistics performance of dairy industries located at Zona da Mata and \\ Campo das Vertentes in Minas Gerais, Brazil
}

\author{
Alexandre Navarro da Silva ${ }^{I}$ João Eustáquio de Lima ${ }^{I I}$ Ronaldo Perez ${ }^{\text {III }}$
}

\section{RESUMO}

Objetivando avaliar a atividade logística nas empresas do setor laticinista atuantes nas regiões Zona da Mata e Campo das Vertentes no estado de Minas Gerais, foram promovidas pesquisas com 40 indústrias que representam o setor avaliado. Os questionários respondidos pela gerência das empresas continha variáveis que possibilitaram caracterizar as empresas quanto à sua produção, logística de captação e distribuição, gestão interna, além de mercado e comercialização. $O$ uso da análise estatística multivariada fatorial foi de grande relevância ao possibilitar a construção de um índice que representou o desempenho logístico de cada uma das empresas, sendo também utilizada para realizar o agrupamento dessas indústrias em 4 grupos que continham características similares. O índice logístico (IL) mostrou que as empresas estudadas da região precisam melhorar as atividades que constituíram sua medida, a fim de tornar os aspectos logísticos um meio de vantagem competitiva. Além disso, os grupos formados também possibilitaram avaliar que não é suficiente atuar na melhoria de apenas uma parte das atividades logísticas, mas sim no conjunto de variáveis que pode influenciar positivamente no resultado final no quesito competitividade e liderança logística.

Palavras-chave: análise estatística multivariada, índice de desempenho, captação de leite, logística de distribuição.

\section{ABSTRACT}

In order to evaluate the logistics activity in the dairy companies operating in Zona da Mata and Campo das Vertentes in Minas Gerais, Brazil a research has been promoted on 40 industries that represent the sector assessed. The questionnaire answered by company employees, contained variables that allowed obtaining information about company identification, gathering and distribution logistics, internal management, marketing and commercialization. Factorial statistical analysis was very important to enable the construction of an index representing the logistics performance of each company and was also used to perform the grouping of these industries into 4 groups containing similar characteristics. The index logistics (IL) showed that companies in the study area still need to improve their activities which were measured to make the logistics a competitive advantage. Moreover, the groups formed also allowed to evaluate that is not enough the improvement at only a part of logistics activities, but is necessary to improve the set of variables that can positively influence the final result in the logistics competitiveness and leadership.

Key words: multivariate statistical analysis, performance index, milk collection, distribution logistics.

\section{INTRODUÇÃO}

Considerando o setor de lácteos, observase a notoriedade desse segmento, representando grande importância à economia do país. Tal setor ocupa o quinto lugar no ranking de maiores produtores do mundo em 2010 e representa 5,3\% da produção mundial (FAO, 2012).

O mundo competitivo vem despertando nas organizações a necessidade de controle mais efetivo do desempenho logístico. Para sobreviver num ambiente de intensa concorrência, a eficiência produtiva tornou-se fator decisivo para a competitividade do setor leiteiro (LOPES, 2007). DEMETER et al. (2009) também destacaram a necessidade de aumento na eficiência, afirmando que a volatilidade dos preços das commodities lácteas na última década forçou o setor a buscar maior eficiência em custos e consolidação no mercado.

\footnotetext{
IDepartamento de Engenharia de Produção e Mecânica, Universidade Federal de Viçosa (UFV), 36570-000, Viçosa, MG, Brasil. E-mail: alexandre.navarro@ufv.br. Autor para correspondência.

IIDepartamento de Economia Rural, UFV, Viçosa, MG, Brasil.

"IIDepartamento de Tecnologia de Alimentos, UFV, Viçosa, MG, Brasil.
} 
Uma grande dificuldade encontrada nesse setor é a elevada variação da produtividade de leite nas fazendas brasileiras, devido à utilização de níveis tecnológicos de produção diferentes ou ainda fatores climáticos, que eleva a sazonalidade da produção, conforme observado por MARIN et al. (2011).

De acordo com QUINLAN et al. (2005), quando o número de produtores diminui e o tamanho destes aumenta, os custos de transporte são consequentemente reduzidos, pois há diminuição no número de rotas de coleta e aumento do volume de carga por caminhão, gerando economia de combustível, de salários com motorista e na manutenção de caminhões.

Dessa forma, um novo desenho da rede logística, o uso de novas ferramentas gerenciais e métodos de alocação de custos de transporte, incluindo decisões relacionadas à granelização da coleta, como capacidade dos caminhões e à tecnologia adotada na coleta de leite, podem contribuir para redução dos custos, contribuindo também para melhoria da qualidade da matéria-prima. Essa integração deve se refletir em termos de custos totais e desempenho operacional do sistema logístico (RENTIZELAS et al., 2008).

De acordo com HAIR JUNIOR et al. (2009), os métodos multivariados de análise de dados têm comprovado amplamente sua eficácia no estudo de grandes massas de informação complexas. Esses métodos são chamados de multidimensionais, pois permitem confrontar duas ou mais variáveis. Podese, então, extrair as tendências mais significativas e hierarquizá-las, reduzindo efeitos que perturbam a percepção global (JOHNSON \& WICHERN, 1992).

Dessa forma, objetiva-se neste estudo, identificar e caracterizar as indústrias laticinistas da região da Zona da Mata e Campo das Vertentes no estado de Minas Gerais, quanto ao desempenho logístico de captação do leite e distribuição dos produtos, por meio da utilização de questionários e tratamento de dados utilizando análise estatística fatorial e de agrupamento.

\section{MATERIAL E MÉTODOS}

Fonte de dados e variáveis utilizadas

Por meio de um diagnóstico, com objetivo de caracterizar a atividade logística na cadeia produtiva do leite nas regiões da Zona da Mata e do Campo das Vertentes em Minas Gerais, foi avaliada a realidade das empresas laticinistas localizadas nessa região, quanto às tecnologias e aspectos logísticos presentes. O diagnóstico foi realizado no período de janeiro a outubro de 2009, numa parceria entre o
Polo de Excelência do Leite e a Universidade Federal de Viçosa (UFV). Foram pesquisadas 40 empresas laticinistas com registro no Serviço de Inspeção Federal (SIF) e com disponibilidade em colaborar com a pesquisa, as quais representam cerca de $70 \%$ das empresas situadas nas regiões analisadas.

A coleta de dados se baseou na utilização de questionário para medir variáveis sobre identificação, logística de captação e distribuição, gestão interna, além de mercado e comercialização. Para o presente estudo, foram avaliadas 15 medidas, as quais foram codificadas de modo a facilitar a apresentação posterior dos resultados e respectivas discussões. As variáveis utilizadas neste estudo foram as seguintes: VLRI - Volume de leite recebido na indústria; NEMP Número de empregados total; VDTK - Densidade de distribuição em volume distribuído/total km; TEXI Tempo de existência da empresa (anos); PUCI - \% de utilização da capacidade instalada; PFPC - \% de frota própria de captação; PFEI - \% de produtos destinados ao comércio estadual e/ou interestadual; LTPE Lead time do pedido (dias); VRPE - Relação volume recebido (L)/número de empregados; VDPE - Volume de produtos distribuídos (kg)/número de empregados; VCTK - Densidade de coleta em volume captado (L)/ total de Km; MLPP - Média de volume de leite por produtor; PPAL - \% de funcionários que trabalham exclusivamente no setor logística; NPRE - Número de produtos registrados pela empresa; PFPD - \% de frota própria de distribuição.

Construção do índice logístico (IL) e grupos de análise Análise fatorial

Foi realizada análise fatorial por meio do modelo representado na Equação 1 (JOHNSON \& WICHERN, 1992).

$$
X=\alpha F+\varepsilon
$$

em que: $X$ - vetor px1 das variáveis observadas; $F$ vetor qx1 das variáveis não-observáveis chamadas de fatores comuns, sendo que $\mathrm{q}<\mathrm{p}$; $\varepsilon$ - vetor $\mathrm{px} 1$ de variáveis aleatórias ou fatores únicos; $\alpha$ - matriz pxq de constantes, chamadas de cargas fatoriais.

Nesse modelo de análise fatorial, pressupõe-se que os fatores comuns sejam ortogonais. Para facilitar a interpretação dos resultados, utilizouse o método de rotação ortogonal varimax, que propõe a rotação dos eixos de referência dos fatores, a fim de redistribuir a variância e atingir um padrão mais simples e significativo (HAIR JUNIOR et al., 2009).

A escolha dos fatores foi realizada por meio da técnica de raiz latente, cujo princípio é de que um fator individual deve explicar a variância de pelo menos uma variável, para que seja mantido na interpretação. Apenas os fatores que apresentam 
autovalores maiores do que 1 (um) são considerados significantes (JOHNSON \& WICHERN, 1992; MANLY, 1986).

A adequação dos dados à análise fatorial foi avaliada pelo teste de esfericidade de Bartlett, que avalia se a matriz de correlações é igual à matriz identidade, ou seja, se as correlações entre as variáveis são iguais a zero. Também foram utilizados os coeficientes MSA (Measure of Sampling Adequacy) e KMO (Kaiser-Meyer-Olkin), que avaliam se as correlações parciais, ou seja, correlações entre as variáveis desconsiderando o efeito das demais variáveis, são iguais a zero (MINGOTI, 2005).

Construção do índice logístico - IL

Para classificar o desempenho logístico das empresas estudas, foram determinados os escores fatoriais pelo método de regressão e definido o Índice Logístico - IL (Equação 2).

$$
I L_{i}=\sum_{j=1}^{q}\left(\frac{\lambda_{j}}{\sum_{j} \lambda_{j}} F P_{i j}\right)
$$

em que: $\lambda$ - variância explicada pelo fator $\mathrm{j}$; FP escore fatorial padronizado.

O escore fatorial foi padronizado (FP) para que fossem obtidos valores positivos dos escores originais e para permitir a classificação das empresas, para que os valores do IL sejam positivos para posterior ordenação e caracterização (Equação 3).

$$
F P_{i j}=\left(\frac{F_{i j}-F_{j \min }}{F_{j \max }-F_{j \min }}\right)
$$

em que: $\mathrm{F}_{\text {jmin }}$ - escore mínimo do fator $\mathrm{j} ; \mathrm{F}_{\mathrm{jmax}}$ - escore máximo do fator $\mathrm{j}$.

Análise de agrupamentos

A análise de agrupamento busca identificar e classificar variáveis ou objetos em grupos distintos, de acordo com determinadas características (MANLY, 1986). Neste estudo, realizou-se o agrupamento por objetos (indústrias).

A análise de agrupamento utiliza o conceito de distância entre as unidades de classificação. Há diversos métodos para mensuração dessa distância, dentre os quais o mais utilizado é a distância euclidiana. Assim, é construída a matriz de distâncias euclidianas para o processo de agrupamento dos objetos. Quanto mais próxima de zero for a distância, maior a similaridade entre os objetos em comparação. Essa distância é expressa algebricamente por:

$$
D_{A B}=\sqrt{\sum_{j=1}^{m}\left(X_{A j}-X_{B j}\right)^{2}}
$$

em que: $\mathrm{D}_{\mathrm{AB}}$ - distância euclidiana do objeto $\mathrm{A}$ ao B; j - indexador das variáveis.

Há diversos métodos disponíveis para combinação dos objetos em grupos, os quais são classificados como hierárquicos e não hierárquicos. Neste estudo, utilizou-se o método sequencial, aglomerativoehierárquicodeWard.Para determinação do número de grupos a serem considerados, não há critério pré-estabelecido, sendo necessária a avaliação crítica dos pesquisadores em cada caso específico. Conforme sugerido por MINGOTI (2005), foi utilizado o histórico de agrupamento pelo método de Ward e avaliado o nível (distância) de agrupamento, sendo estabelecido o ponto de corte, de modo a possibilitar um número de grupos gerenciável e que permitisse interpretação prática.

Posteriormente, para caracterizar os grupos em função das variáveis medidas, realizou-se teste de comparações pareadas de Bonferroni para os grupos encontrados. O teste foi escolhido pela necessidade de comparar grupos com números de observações desbalanceados.

As análises foram realizadas utilizando o software estatístico Stata 12.0 .

\section{RESULTADOS E DISCUSSÃO}

\section{Obtenção e caracterização dos fatores}

Realizando o teste de esfericidade de Bartlett, rejeita-se (P-valor $<0,001)$ a hipótese de que a matriz de correlações entre as variáveis seja igual à matriz identidade, portanto os dados podem ser adequados à realização da análise fatorial. Ao obter os coeficientes MSA (Measure of Sampling Adequacy) e KMO (Kaiser-Meyer-Olkin), verifica-se que algumas variáveis apresentam baixo coeficiente MSA, o que leva a obter reduzido valor para $\operatorname{KMO}(0,4683)$, expondo a adequabilidade reduzida dos dados à realização da análise fatorial. Porém, os resultados obtidos por meio desses testes não inviabilizam a utilização da técnica escolhida, pois, apesar do reduzido valor de KMO, há alta significância do teste de esfericidade de Bartlett.

Pelo critério da raiz latente, 6 fatores (F1 a F6) foram considerados significantes na análise, explicando $72,70 \%$ da variação inerente aos dados (Tabela 1). As comunalidades das variáveis se apresentam com valores elevados, sendo um indicativo de que a análise permitiu a captação de grande parte da variabilidade associada a cada uma das variáveis observadas. As cargas fatoriais permitem definir quais variáveis participam com maior peso em cada fator (valores acima de 0,55), conforme 
Tabela 1 - Cargas fatoriais, comunalidades das variáveis estudadas e percentual de explicação de cada autovalor relacionado aos fatores.

\begin{tabular}{lllllll}
\hline Variável & F1 & F2 & F3 & F4 & F5 & F6 \\
\hline VLRI & $0,937^{*}$ & $-0,082$ & 0,110 & $-0,059$ & $-0,056$ & 0,126 \\
NEMP & $0,818^{*}$ & $-0,074$ & $-0,212$ & $-0,098$ & $-0,094$ & 0,311 \\
VDTK & $0,803^{*}$ & 0,093 & 0,438 & $-0,032$ & 0,034 & $-0,005$ \\
TEXI & $0,594^{*}$ & 0,100 & $-0,006$ & 0,523 & $-0,051$ & $-0,126$ \\
PUCI & $0,579^{*}$ & $-0,046$ & $-0,031$ & 0,495 & $-0,100$ & $-0,174$ \\
PFPC & 0,025 & $0,767^{*}$ & $-0,271$ & $-0,031$ & $-0,082$ & 0,034 \\
PFEI & 0,150 & $-0,736^{*}$ & $-0,059$ & $-0,081$ & $-0,259$ & $-0,111$ \\
LTPE & 0,015 & $-0,633^{*}$ & $-0,158$ & $-0,069$ & 0,522 & 0,096 \\
VRPE & 0,147 & 0,018 & $0,778^{*}$ & $-0,056$ & 0,202 & $-0,179$ \\
VDPE & 0,167 & $-0,297$ & $0,628^{*}$ & 0,010 & $-0,188$ & 0,244 \\
VCTK & $-0,135$ & 0,286 & $-0,144$ & $0,650^{*}$ & $-0,085$ & 0,624 \\
MLPP & 0,194 & 0,223 & $-0,072$ & $-0,574 *$ & $-0,371$ & 0,671 \\
PFPD & 0,223 & 0,429 & $-0,409$ & $-0,432^{*}$ & 0,273 & 0,260 \\
PPAL & $-0,109$ & 0,054 & 0,088 & $-0,008$ & $0,868^{*}$ & $-0,288$ \\
NPRE & 0,217 & 0,029 & $-0,018$ & 0,006 & 0,118 & 0,114 \\
Autovalor & 3,117 & 1,971 & 1,554 & 1,488 & 1,434 & 0,704 \\
Explicação & 0,208 & 0,131 & 0,104 & 0,099 & 0,096 & 0,633 \\
\hline
\end{tabular}

*Variáveis de maior peso nos respectivos fatores.

destacadas com asterisco na tabela 1 . Assim, o primeiro fator é destacado por representar o tempo de existência, número de empregados, percentual de utilização da capacidade instalada, volume de recepção e densidade de distribuição de produtos acabados. A interpretação para os outros fatores é semelhante.

Dessa forma, as variáveis que apresentam maiores escores relacionados aos fatores F1 e F2 tendem a ser mais representativas do conjunto de variáveis estudado, um vez que estas representam maior variação dos dados frente às demais. Assim, as seguintes variáveis devem ser melhor controladas para que o desempenho logístico da empresa seja melhorado: tempo de existência (TEXI), número de empregados (NEMP), percentual de utilização da capacidade instalada (PUCI), volume de recepção (VLRI), densidade de distribuição de produtos acabados (VDTK), percentual de frota própria de captação (PFPC), percentual de distribuição estadual-interestadual (PFEI) e lead time de entrega (LTPE).

Índice Logístico - IL

Com base nos escores fatoriais padronizados, foi possível a construção do Índice Logístico (IL), que representa o desempenho logístico das empresas estudadas, sendo representados na tabela 2 os valores para o IL, aliados à respectiva carga fatorial padronizada para cada indústria.

Uma vez que este índice possui amplitude de variação compreendida entre zero e um, afirma- se que a qualidade logística das empresas avaliadas é baixa, uma vez que o maior IL obtido foi o valor 0,563, sendo que o índice médio foi de 0,311, com desvio-padrão de 0,087 .

Observa-se que as empresas com maior valor de IL apresentam escores fatoriais elevados para os primeiros fatores, os quais representam maior variação dos dados, sendo, portanto, de maior importância para explicação do fenômeno estudado, que é a variabilidade de desempenho logístico entre as empresas laticinistas. Já as empresas com menor IL apresentam baixo escore fatorial para todos os fatores, demonstrando valores reduzidos para as variáveis representantes dos fatores, as quais são indicadores de desempenho logístico. Esse resultado corrobora o exposto anteriormente, sendo que as 8 variáveis relacionadas com os fatores F1 e F2 devem ser controladas e gerenciadas de forma a garantir a qualidade logística das indústrias laticinistas das regiões estudadas.

Obtenção e caracterização dos grupos de indústrias

Com base nos fatores definidos, realizou-se a análise de agrupamento, a fim de estabelecer um baixo número de grupos de empresas, em que há grande similaridade dentro de cada grupo e diferenças entre os grupos. Essa técnica facilita o gerenciamento da informação, uma vez que se pode generalizar a tomada de decisão para os grupos, não sendo necessária a construção de políticas e diretrizes para cada 
Tabela 2 - Cinco menores e cinco maiores índices com os respectivos fatores padronizados. Destacam-se os fatores mais representativos para cada empresa.

\begin{tabular}{|c|c|c|c|c|c|c|c|}
\hline \multirow{2}{*}{ Empresa } & & \multicolumn{5}{|c|}{ 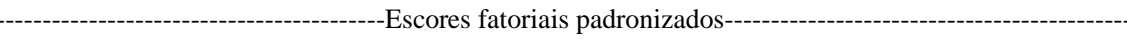 } & \multirow{2}{*}{ IL } \\
\hline & $\mathrm{F} 1$ & $\mathrm{~F} 2$ & F3 & F4 & F5 & F6 & \\
\hline 24 & 0.108 & 0.092 & 0.196 & 0.424 & 0.278 & 0.134 & 0.187 \\
\hline 40 & 0.037 & 0.275 & 0.302 & 0.353 & 0.154 & 0.199 & 0.196 \\
\hline 15 & 0.126 & 0.189 & 0.227 & 0.397 & 0.149 & 0.195 & 0.200 \\
\hline 10 & 0.000 & 0.307 & 0.265 & 0.353 & 0.251 & 0.258 & 0.206 \\
\hline 39 & 0.102 & 0.351 & 0.255 & 0.258 & 0.295 & 0.143 & 0.221 \\
\hline 22 & 0.159 & 0.400 & 0.145 & 0.180 & 0.298 & 0.170 & 0.223 \\
\hline 19 & 0.140 & 0.298 & 0.193 & 0.279 & 0.426 & 0.088 & 0.226 \\
\hline 25 & 0.157 & 0.388 & 0.211 & 0.314 & 0.229 & 0.102 & 0.231 \\
\hline 27 & 0.233 & 0.305 & 0.250 & 0.304 & 0.245 & 0.090 & 0.242 \\
\hline 32 & 0.364 & 0.039 & 0.012 & 0.333 & $0.518 *$ & 0.142 & 0.244 \\
\hline 28 & 0.158 & $0.551 *$ & 0.122 & 0.282 & 0.274 & 0.073 & 0.246 \\
\hline 18 & 0.121 & 0.297 & 0.396 & $0.503 *$ & 0.162 & 0.159 & 0.254 \\
\hline 31 & 0.210 & 0.000 & 0.060 & 0.265 & $1.000 *$ & 0.164 & 0.256 \\
\hline 23 & 0.250 & $0.520^{*}$ & 0.129 & 0.239 & 0.187 & 0.143 & 0.259 \\
\hline 38 & 0.304 & 0.206 & 0.219 & $0.523^{*}$ & 0.200 & 0.108 & 0.266 \\
\hline 34 & 0.369 & $0.623^{*}$ & 0.213 & 0.000 & 0.000 & 0.243 & 0.278 \\
\hline 33 & 0.164 & 0.220 & 0.346 & $0.559 *$ & 0.413 & 0.112 & 0.280 \\
\hline 37 & 0.189 & 0.329 & 0.166 & 0.174 & 0.427 & $0.526^{*}$ & 0.282 \\
\hline 1 & 0.209 & 0.379 & 0.193 & 0.382 & 0.387 & 0.238 & 0.288 \\
\hline 2 & 0.256 & 0.249 & 0.125 & 0.332 & $0.681^{*}$ & 0.172 & 0.292 \\
\hline 26 & 0.316 & 0.193 & 0.280 & 0.425 & 0.365 & 0.183 & 0.293 \\
\hline 9 & 0.239 & 0.302 & 0.173 & $0.572^{*}$ & 0.390 & 0.190 & 0.300 \\
\hline 29 & 0.490 & 0.157 & 0.074 & 0.462 & 0.343 & 0.220 & 0.314 \\
\hline 14 & 0.102 & 0.983* & 0.233 & 0.193 & 0.228 & 0.231 & 0.325 \\
\hline 20 & 0.092 & $0.884 *$ & 0.240 & 0.325 & 0.390 & 0.144 & 0.334 \\
\hline 30 & 0.244 & $0.591 *$ & 0.221 & 0.396 & $0.518 *$ & 0.064 & 0.338 \\
\hline 8 & 0.295 & 0.492 & 0.133 & $0.587 *$ & 0.402 & 0.119 & 0.340 \\
\hline 13 & 0.355 & 0.164 & 0.285 & 0.388 & 0.329 & $0.592 *$ & 0.341 \\
\hline 12 & 0.470 & $0.534 *$ & 0.150 & 0.455 & 0.228 & 0.000 & 0.344 \\
\hline 21 & 0.169 & 0.317 & 0.330 & 0.213 & 0.306 & $1.000 *$ & 0.345 \\
\hline 36 & 0.401 & 0.029 & $0.586 *$ & 0.455 & 0.143 & 0.492 & 0.345 \\
\hline 17 & 0.206 & $0.971^{*}$ & 0.273 & 0.230 & 0.188 & 0.127 & 0.345 \\
\hline 6 & 0.388 & $0.554 *$ & 0.214 & 0.455 & 0.390 & 0.024 & 0.358 \\
\hline 4 & 0.320 & $0.678^{*}$ & 0.260 & $0.609 *$ & 0.293 & 0.169 & 0.394 \\
\hline 5 & 0.225 & 0.404 & $1.000^{*}$ & 0.291 & $0.593^{*}$ & 0.089 & 0.409 \\
\hline 16 & $0.781^{*}$ & $0.560 *$ & 0.000 & 0.199 & 0.213 & 0.438 & 0.434 \\
\hline 3 & 0.351 & $0.893^{*}$ & 0.400 & 0.422 & 0.542 & 0.023 & 0.451 \\
\hline 11 & 0.144 & $0.872 *$ & 0.107 & $1.000 *$ & 0.240 & $0.614^{*}$ & 0.458 \\
\hline 35 & 0.198 & $1.000^{*}$ & 0.238 & 0.340 & $0.971^{*}$ & $0.562 *$ & 0.515 \\
\hline 7 & $1.000^{*}$ & 0.489 & 0.459 & 0.377 & 0.393 & 0.167 & 0.563 \\
\hline
\end{tabular}

*Escores fatoriais de maior relevância para composição do IL de cada empresa.

empresa separadamente, mas sim para grupos de empresas pré-estabelecidos.

As dissimilaridades entre as indústrias foram representadas com dendrogramas pela figura 1A, sendo também representado o nível de referência considerado, uma vez que 4 grupos foram definidos (Figura 1B). Os grupos foram caracterizados pelas variáveis estudadas. A tabela 3 demonstra as médias dessas medidas para cada grupo e o teste de Bonferroni aplicado entre os grupos.

As empresas constituintes do grupo 1 diferem dos demais grupos por apresentar Índice Logístico estatisticamente inferior aos demais, com valor médio de 0,24. Apesar de os grupos 2, 3 e 4 não 


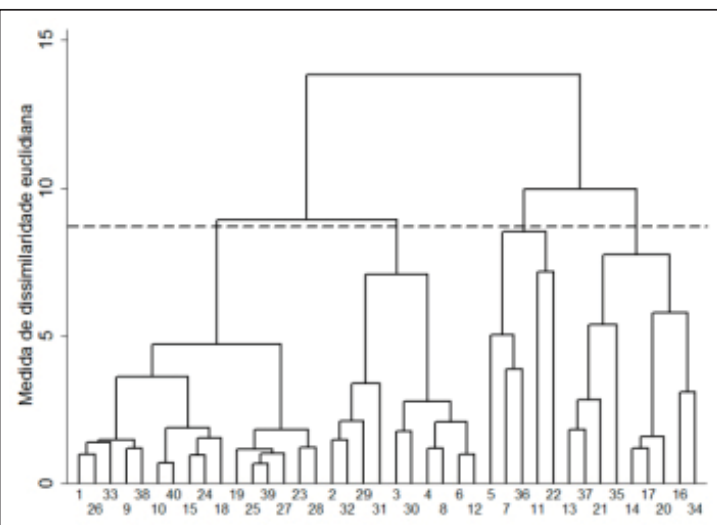

(A)

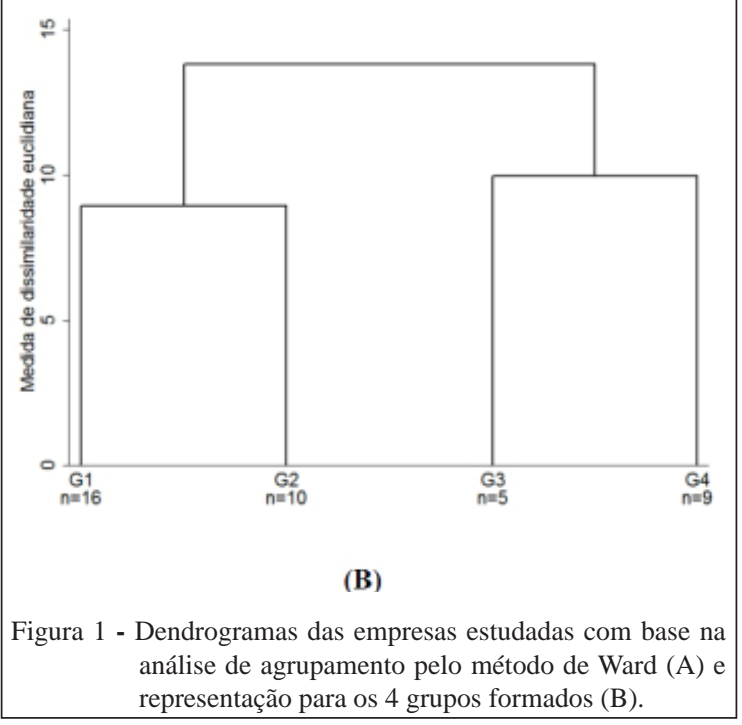

apresentarem diferença significativa entre os valores médios de IL, isso não significa que as características desses grupos são as mesmas, ou seja, a diferenciação entre os grupos é devida às especificidades definidas pelas variáveis medidas.

O grupo 1, constituído de 16 empresas, é caracterizado por apresentar valores inferiores aos demais grupos para todas as medidas, sendo constituído de empresas com médio tempo de existência (18 anos), poucos empregados (27) e volume de recepção (15.000L) e também baixo percentual de frota própria para coleta de leite (14\%) e distribuição dos produtos para o mercado (43\%), que é predominantemente estadual e interestadual (93\%).

Para construção do grupo 2, foram concentradas 10 indústrias, caracterizadas por apresentar elevado tempo de existência (37 anos), número intermediário de empregados (63) e volume de recepção (34.000L), sendo grande o percentual de pessoas na área de logística (13\%), tendo mercado consumidor predominantemente em termos estaduais e interestaduais (82\%) e grande percentual de frota própria para distribuição dos produtos (85\%).

As cinco empresas constituintes do grupo 3 são estabelecidas no mercado há bastante tempo (35 anos), apresentando índices elevados de produtividade, quando avaliadas variáveis de volume de recepção por número de empregados (1640L pessoa $\left.^{-1}\right)$, densidade de coleta de leite $\left(543 \mathrm{~L} \mathrm{~km}{ }^{-1}\right.$ ) e distribuição de produtos $\left(586 \mathrm{~L} \mathrm{~km}^{-1}\right)$, mesmo com baixo percentual de pessoas na área de logística (4\%). Para esse grupo, o mercado consumidor também está localizado por grande parte do estado e também em outros estados do país (86\%). Destaca-se que as empresas que fazem parte desse grupo são as maiores indústrias avaliadas.

No grupo 4, encontram-se empresas com menor tempo de existência (16 anos), porém que apresentam IL $(0,36)$ semelhante às empresas dos grupos $2(0,33)$ e $3(0,40)$, que possuem maior tempo no mercado. Caracterizado por apresentar maior volume de leite médio por produtor (259L produtor $^{-1}$ ) e grande percentual de frota própria de captação (58\%) e distribuição de produtos (78\%), esse grupo possui menor amplitude de distribuição dos produtos acabados, mantendo-se em grande percentual no mercado local e regional (42\%).

A estratégia de mercado e comercialização dos produtos deve ser traçada com a preocupação referente à forma de captação de leite dos produtores. Assim, a distribuição estadual-interestadual prejudica a qualidade logística das empresas laticinistas das regiões avaliadas, uma vez que estas não possuem estrutura suficiente para garantir bom atendimento aos clientes em maiores distâncias, sendo que o maior desempenho seria alcançado para distribuição local e regional dos produtos.

A disposição dos grupos mostra que, além das variáveis relacionadas aos dois primeiros fatores, também deve ser observada a eficiência de utilização dos recursos humanos pela empresa, ou seja, empresa com melhor IL (Grupo 3) também apresenta maior volume recebido e distribuído por número de empregados, variáveis estas que caracterizam o fator F3.

\section{CONCLUSÃO}

A caracterização das atividades logísticas do setor laticinista das regiões da Zona da Mata e dos Campos das Vertentes em Minas Gerais mostrou que dez entre as 15 variáveis analisadas são de maior importância para captar a qualidade logística 
Tabela 3 - Médias das variáveis para cada um dos grupos formados pela análise de agrupamento e teste de médias de Bonferroni.

\begin{tabular}{|c|c|c|c|c|c|}
\hline \multirow{2}{*}{ Variáveis } & \multicolumn{4}{|c|}{ 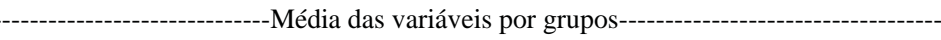 } & \multirow{2}{*}{ Média Global } \\
\hline & Grupo 1 & Grupo 2 & Grupo 3 & Grupo 4 & \\
\hline VLRI & $15050^{c}$ & $34000^{\mathrm{b}}$ & $70800^{a}$ & $40777^{\mathrm{b}}$ & 32545 \\
\hline NEMP & $26,75^{\mathrm{b}}$ & $63,10^{\mathrm{a}, \mathrm{b}}$ & $77,40^{\mathrm{a}, \mathrm{b}}$ & $95,10^{a}$ & 57,55 \\
\hline VDTK & $30,19^{b}$ & $172,66^{\mathrm{b}}$ & $586,44^{a}$ & $133,18^{\mathrm{b}}$ & 158,51 \\
\hline TEXI & $18,80^{\mathrm{b}}$ & $37,60^{a}$ & $35,00^{a}$ & $16,60^{\mathrm{b}}$ & 25,00 \\
\hline PUCI & $0,58^{\mathrm{a}}$ & $0,77^{\mathrm{a}}$ & $0,64^{\mathrm{a}}$ & $0,51^{\mathrm{a}}$ & 0,62 \\
\hline PFPC & $0,14^{\mathrm{b}}$ & $0,33^{\mathrm{a}, \mathrm{b}}$ & $0,34^{\mathrm{a}, \mathrm{b}}$ & $0,58^{a}$ & 0,31 \\
\hline PFEI & $0,93^{a}$ & $0,82^{a}$ & $0,86^{a}$ & $0,58^{\mathrm{b}}$ & 0,81 \\
\hline LTPE & $3,00^{\mathrm{a}}$ & $4,10^{\mathrm{a}}$ & $4,00^{\mathrm{a}}$ & $2,40^{\mathrm{a}}$ & 3,28 \\
\hline VRPE & $406,24^{\mathrm{b}}$ & $629,21^{\mathrm{b}}$ & $1640,56^{a}$ & $445,43^{b}$ & 625,09 \\
\hline VDPE & $206,55^{\mathrm{b}}$ & $75,57^{\mathrm{b}}$ & $721,68^{a}$ & $187,86^{\mathrm{b}}$ & 233,99 \\
\hline VCTK & $89,29^{b}$ & $102,34^{\mathrm{b}}$ & $543,34^{a}$ & $59,11^{\mathrm{b}}$ & 142,52 \\
\hline MLPP & $113,30^{\mathrm{b}}$ & $110,99^{\mathrm{b}}$ & $133,74^{\mathrm{b}}$ & $259,42^{a}$ & 148,16 \\
\hline PPAL & $0,04^{\mathrm{b}}$ & $0,13^{a}$ & $0,04^{\mathrm{b}}$ & $0,09^{\mathrm{a}, \mathrm{b}}$ & 0,08 \\
\hline NPRE & $9,00^{\mathrm{b}}$ & $16,40^{\mathrm{b}}$ & $27,4,0^{\mathrm{a}, \mathrm{b}}$ & $47,67^{a}$ & 21,85 \\
\hline PFPD & $0,43^{\mathrm{b}}$ & $0,85^{a}$ & $0,44^{\mathrm{b}}$ & $0,78^{a}$ & 0,62 \\
\hline Índice Logístico IL & $0,24^{\mathrm{b}}$ & $0,33^{\mathrm{a}, \mathrm{b}}$ & $0,40^{a}$ & $0,36^{a}$ & 0,31 \\
\hline
\end{tabular}

Médias seguidas de mesma letra (a, b, c) na linha não diferem entre si ao nível de significância de 10\%, pelo teste de Bonferroni. Itálico indica maior média para cada variável.

das indústrias. Resultado este sugerido pela análise fatorial e corroborado pelo índice logístico e também pelo agrupamento das empresas, realizado de acordo com as variáveis originais.

Ao gerenciar o desempenho das variáveis que apresentam maiores escores relacionados aos fatores F1, F2 e F3, as empresas apresentarão a tendência de se destacarem em relação às atividades logísticas frente às demais. Assim, as seguintes variáveis devem ser melhor controladas para que o desempenho logístico da empresa seja melhorado: tempo de existência (TEXI), número de empregados (NEMP), percentual de utilização da capacidade instalada (PUCI), volume de recepção (VLRI), densidade de distribuição de produtos acabados (VDTK), percentual de frota própria de captação (PFPC), percentual de distribuição estadual-interestadual (PFEI), lead time de entrega (LTPE), volume recebido por número de empregado (VRPE) e volume distribuído por número de empregado (VDPE).

\section{REFERÊNCIAS}

DEMETER, R.M. et al. Scenarios for a future dairy chain in the Netherlands. NJAS- Wageningen Journal of Life Sciences, v.56, n.4, p.301-322, 2009. Disponível em: <http://dx.doi.org/10.1016/ S1573-5214(09)80002-X>. Acesso em: 15 jun. 2012. doi: 10.1016/S1573-5214(09)80002-X.

FOOD AND AGRICULTURAL ORGANIZATION (FAO). Livestock primary - Cow milk. Disponível em: <http://faostat. fao.org/>. Acesso em: 25 jun. 2012.
HAIR JUNIOR, J.F. et al. Multivariate data analysis. 7.ed. New Jersey: Prentice Hall, 2009. 816 p.

JOHNSON, R.A.; WICHERN, D.W. Applied multivariate statistical analysis. 3.ed. Englewood Cliffs: Prentice Hall, 1992. 800 p.

LOPES, P.F. Custos e escala de produção na pecuária leiteira: estudo nos principais estados produtores do Brasil. Revista de Economia e Sociologia Rural, v.45, n.3, p. 567-590, 2007. Disponível em: $<$ http://dx.doi.org/10.1590/S0103-20032007000300002>. Acesso em: 01 abr. 2012. doi: 10.1590/S0103-20032007000300002.

MANLY, B.F.J. Multivariate statistical methods - a primer. New York: Chapman and Hall, 1986. 159 p.

MARIN, S.R. et al. Sazonalidade do preço do leite no Rio Grande do Sul (1986-2009). Ciência Rural, v.41, n.2, p.361364, 2011. Disponível em: <http://dx.doi.org/10.1590/S010384782011000200030>. Acesso em: 13 maio, 2012. doi: 10.1590/ S0103-84782011000200030.

MINGOTI, S.A. Análise de dados através de métodos de estatística multivariada: uma abordagem aplicada. Belo Horizonte: UFMG, 2005. 295 p.

QUINLAN, C. et al. The cost and efficiency of milk transport from farms in Ireland. Department of Food Business and Development University College, Cork - Ireland. Agribusiness Discussion Paper, n.46, p.1-30, 2005. Disponível em: <http://www.ucc.ie/en/ foodbus/research/discussion/fortyonetofifty/DocumentFile-32510en.pdf>. Acesso em: 23 maio, 2012.

RENTIZELAS, A.A. et al. Logistics issues of biomass: the storage problem and the multi-biomass supply chain. Renewable and Sustainable Energy Reviews, v.13, p 887-894, 2008. Disponível em: <http://dx.doi.org/10.1016/j.rser.2008.01.003>. Acesso em: 10 maio, 2012. doi: 10.1016/j.rser.2008.01.003. 